\title{
Measuring fluid intelligence on a ratio scale: Evidence from nonverbal classification problems and information entropy
}

\author{
PhiLippe E. Ruiz \\ ESC Lille Graduate School-LSMRC, Euralille, France
}

\begin{abstract}
Drawing on the $g$ factor and information theory literatures, the relationship between the four subtests of the Culture-Fair Intelligence Test (CFIT) and the entropy of the Ruiz Absolute Scale of Complexity Management (R-ASCM) was investigated. In results based on data collected from 186 university students, the entropy of the R-ASCM mostly loads the first principal component extracted from the CFIT subtests and shows a corresponding strong relationship with the item difficulty of the R-ASCM. Because entropy is a ratio scale of complexitywith a true zero and units called bits - these findings suggest that entropy is the right vehicle for measuring the information contained in nonverbal intelligence tests.
\end{abstract}

The psychometric concept of intelligence was mostly the invention of Charles Spearman (1904). Its value has been demonstrated in numerous studies. General intelligence - also known as the $g$ factor-is a wideranging tendency for those who are the best at one thing to be the best at others (human abilities are said to be positively correlated).

An influential theory of the structure of intelligence was proposed by Cattell $(1941,1971)$ and extended by Horn (1968, 1985). Cattell and Horn identified two major components, which they labeled fluid intelligence $\left(g_{\mathrm{f}}\right)$ and crystallized intelligence $\left(g_{\mathrm{c}}\right)$. Fluid intelligence is a largely nonverbal form of mental efficiency related to a person's capacity to learn and solve new problems. By contrast, crystallized intelligence represents what one has already learned through the investment of fluid intelligence in cultural settings. The present study contributes to knowledge about fluid intelligence, what it reflects, and how it can be better measured on a ratio scale.

\section{LITERATURE REVIEW AND HYPOTHESIS}

The Culture-Fair Intelligence Test (Cattell, 1940; IPAT, 1973a, 1973b) is a nonverbal measure of fluid intelligence used in this research. Cattell introduced his test, first published in 1940, in the Journal of Educational Psychology:

In general the kinds of test showing the best " $G$ " saturation are those involving relation and correlate eduction in a high degree and reproduction in the lowest degree. The individual's general ability might, therefore, be defined by the order of complexity of the relations which he is capable of handling. (p. 164)
The $g$ factor is essentially the ability to deal with cognitive complexity; in particular, complex information processing (Gottfredson, 1997). The more complex a task, the greater the advantages that higher $g$ confers in performing it well. The important element in mental tests is their complexity or the amount of conscious mental manipulation they require (Jensen, 1980), not surface traits or specific indicators such as pictures, words, symbols, numbers, and so on, as originally noted by Spearman (1923).

There is a long history of the study of simplicity in psychology, and a shorter one of complexity. Applications of the notion of simplicity, not always its polar opposite, were pioneered by Hochberg and McAlister (1953), particularly in the field of perception. As noted by Goodman (1972), all solidly based approaches to simplicity or complexity involve counting primitives. Campbell (1988) has studied the nature of objective task complexity in the fields of information processing and decision making. The various definitions he has identified emphasize the number, variability, variety, and interrelatedness of information to be processed.

Relational complexity (Halford, Wilson, \& Phillips, 1998 ) is related to processing loads in problem solving, and discriminates between higher animal species, as well as between children of different ages. Complexity in this context is defined by the number of dimensions, or sources of variation, that are related. More recently, the conceptual complexity of problems was manipulated to probe the limits of human information processing capacity (Halford, Baker, McCredden, \& Bain, 2005). Subjects were asked to interpret graphically displayed statistical interactions. These findings suggest that a structure defined on four variables is at the limit of human processing

P. E. Ruiz, p.ruiz@esc-lille.fr 
capacity. Assessment of processing capacity is difficult because of the great power of mental strategies for reducing processing load, thereby optimizing use of available capacity.

The latter work offers a ratio scale of measurement for the number of variables that the human brain can process. Undeniably, research in all areas of science is dependent on adequate measurement methods. According to Stevens (1946) and Narens (1985), all numbers derived from measurement instruments of any type can be placed into one of five hierarchical categories: nominal, ordinal, interval, ratio, and absolute. In nominal scales, the numbers serve only as category names. Ordinal scales constitute a form of ordering or ranking. Interval scales provide information about ranking, but also supply a metric for gauging the differences between rankings; they possess equal intervals. The most satisfactory type of measuring instrument for scientific purposes, however, is a ratio scale, which has a true zero, and in which the units of measurement are at equal intervals throughout. Finally, an absolute scale is a special type of ratio scale for which there is one and only one way to map the objects into numbers-for example, the total number of words in this paper.

Ratio scales are rare in the cognitive sphere, mostly because norm-referenced tests constitute the vast majority of measurement methods currently in use. In a normreferenced instrument, the performance of each examinee is interpreted with reference to a relevant standardization sample (Petersen, Kolen, \& Hoover, 1989). Barrett (2000) describes the limitations of the scales used by Cattell and Horn and concludes that there are no units of " $g$," no units of "IQ," and no units of "intelligence." The scores on psychometric tests represent an ordinal scale, merely ranking individuals according to their overall performance on a variety of complex mental tasks; this deficiency obstructs the development of a true natural science of mental ability (Jensen, 2006). The reliability and, therefore, the validity of intelligence tests from one generation to the next has been questioned by Flynn (1987). Wicherts et al. (2004) found further evidence from five data sets that IQ scores are not measurement invariant but structurally different over time.

For Heylighen (1994), survival in a variable environment requires an internal model whose complexity matches the complexity of the environment to be controlled; also, the reduction of the sensed environment to an adequate map requires a strong mechanism of categorization. He further defines a measure of cognitive complexity as the statistical entropy of the system under consideration.

Information theory (IT) is a foundational subject for computer scientists, engineers, statisticians, biologists, and cognitive scientists alike. In information theory, the statistical entropy or information entropy is a measure of the uncertainty associated with a random variable. Entropy quantifies the information contained in a message or system, usually in bits. Information here does not refer to any specific fact or a particular item of knowledge, but to any stimulus that reduces uncertainty. A bit (binary digit) is a digit in the binary numeral system and it can hold only one of two values: 0 or 1 . Information can thus be defined in the statistical sense, as the degree to which data are noncompressible. Consequently, and to avoid any confusion, "information" in the present study is defined by Shannon's (1948) original use in information theory, that of statistical information, also known as entropy.

Shannon (1948) defines entropy as an $\mathrm{H}$ function of the following variety:

$$
H=-K \sum_{i=1}^{k} p(i) \log p(i),
$$

where $K$ is a positive constant often equal to $1, k$ the number of different elements in the system, $p(i)$ the probability of occurrence of one particular element $i$ and $\log p(i)$ the quantity of information necessary to represent such an element (base 2 or bits are generally used for that purpose). Shannon then explains that any quantity of this form plays a central role in information theory as a measure of information, choice, and uncertainty.

Entropy is of fundamental importance, because it captures holistically the total number of elements inside a system as well as their precise information content in bits weighted by their respective frequency. Above all, because entropy has a true zero and equal intervals, it constitutes a ratio scale.

To summarize as a hypothesis: Information entropy is a measure of complexity that can measure fluid intelligence on a ratio scale.

\section{METHOD}

\section{Sample}

We assessed 186 students of a top-flight French business school, the École Supérieure de Commerce (ESC) Lille School of Management; all of them provided usable responses. Participation was voluntary and was obtained from a general announcement via the school e-mail system addressing all graduates and undergraduates. Research data were collected at one point in time during a single testing session on the school campus. There were 105 females (56\%) and 81 males $(44 \%)$. All were native or fluent French speakers, mostly of Caucasian origin. The average age of the students was 22.4 years $(S D=3.2)$.

\section{Measures}

Culture-Fair Intelligence Test (CFIT): Scale 3, Form B. The CFIT (Cattell, 1940; IPAT, 1973a, 1973b) is a nonverbal measure of fluid intelligence suitable for age 4 through adult. It comes in three versions or scales, each consisting of two equivalent forms (A and B). Each form consists of four subtests: series, classification, matrices, and conditions. It was decided to use Scale 3, which is for high-ability adults and high-school and college students. Form B, which is referred to as a short test (whereas both forms together are called a full test), was used alone. The four subtests of Form B are each highly speeded, with time limits varying from 2.5 to $4 \mathrm{~min}$. The subject's total working time is only 12.5 min for 50 questions, but the test itself takes about $30 \mathrm{~min}$ to give.

The CFIT is considered an excellent brief and nonverbal measure of general intelligence. The validity of the CFIT as a measure of general intelligence has been established beyond a reasonable doubt (Gregory, 2004). CFIT scores correlate in the mid-.80s with the general factor of intelligence and show consistently robust relationships, mostly in the $.70 \mathrm{~s}$ and $.80 \mathrm{~s}$, with other mainstream measures of intelligence: WISC, WAIS, Raven's Progressive Matrices, Stanford-Binet, Otis, and General Aptitude Test battery (see IPAT, 1973a, p. 11).

A major advantage of the CFIT is that it contains a subtest named classification, in which for each question the subjects must pick out 
two odd items in each row of figures. This oddity measure is quite similar to the one obtained from the R-ASCM described below.

Ruiz Absolute Scale of Complexity Management (R-ASCM) Standard, Form A. The R-ASCM is an assessment instrument that comes in two versions: standard (Forms A and B) and advanced. Each standard form and advanced version consists of 35 discrete oddity problems of increasing difficulty (see Figure 1). Each standard form takes $15 \mathrm{~min}$ to administer, whereas the advanced version takes $1 \mathrm{~h}$ to complete (or can be administered with no time limit as a power test). The standard version, Form A was used. The subjects were given five practice problems, then were instructed to find the odd man out for the 35 questions.

The R-ASCM questions were constructed from elements as easy to identify as possible: squares, circles, crosses, triangles, and the like. The elements constitute the fundamental content of the test questions. What is unique here is that the elements are discrete symbols of equal importance - they can easily be counted for quantification purposes. For each problem in the test, the subjects find cells filled with symbols of no particular significance - say, a triangle, a circle, and a star. All the cells in a question contain the same number of symbols. The shape of these symbols, or their position inside the cells, is of no importance. Only their presence or absence in a cell matters. This test is completely nonverbal, but is not spatial or geometrical. Symmetries or rotations play no part in it. The purpose of every question is to find the cell that is different from all the others. This cell exists and is unique. To find the correct solutions, the subjects must analyze (break down at the symbol level), synthesize (build sets), and judge (critically evaluate their options).

There is virtually no limit to the variety and complexity of problems that can be created with this oddity format (e.g., it is possible to create a problem consisting of 1,000 cells, each filled with 1,000 symbols). After considerable practice with the discrete oddity problem format, it is difficult to believe that the set of all problems can be built in a systematic way, except maybe at the easiest level. This absence of obvious heuristic is most likely why the R-ASCM is a good instrument for measuring fluid intelligence.

R-ASCM entropy. The traditional raw scores, which have at best interval scale properties, must be replaced by entropy scores in bits. Entropy, as noted above, is measured on a ratio scale: It has a true zero and equal intervals. It is impossible to obtain entropy scores from the CFIT or from any other major intelligence test currently available, precisely why the R-ASCM was developed. Entropy (see Formula 1) is first calculated for every R-ASCM question. The R-ASCM can actually be broken down into its basic components. Since the main principle underlying the discrete oddity problem format of the R-ASCM is the inclusion or exclusion of some distinct symbols, the interactions between these symbols do not contribute to the complexity of the test, hence allowing the direct measurement of item complexity with entropy. Entropy is calculated for every R-ASCM question from the symbols themselves. Entropy increases with the total number of symbols, their variety (number of different symbols), and with the symbols' respective frequencies; equal frequencies will increase entropy, whereas unequal frequencies will decrease it.

Let us explain some calculations in detail. For Example 1 in Figure 1 , we write: $\mathrm{H}=-2 / 3 \log _{2}(2 / 3)-1 / 3 \log _{2}(1 / 3)=0.92$ bits Because we have three triangles in the first cell, three triangles in the second cell, and three crosses in the third cell, we have a total of nine symbols: $6 / 9$ are triangles and $3 / 9$ are crosses, or $2 / 3$ and $1 / 3$ respectively - hence the calculation above.

The second calculation should clarify things further. For Example 4 (Figure 1), we get: $\mathrm{H}=-7 / 12 \log _{2}(7 / 12)-1 / 12 \log _{2}(1 / 12)-$ $1 / 12 \log _{2}(1 / 12)-1 / 12 \log _{2}(1 / 12)-1 / 12 \log _{2}(1 / 12)-$ $1 / 12 \log _{2}(1 / 12)=1.95$ bits. We have a total of 12 symbols, 7 of which are arrows, 1 is a cross, 1 is a triangle, 1 is a circle, 1 is a kind of $\mathrm{S}$, and 1 is a kind of $\mathrm{N}$. The seven arrows give an entropy of $-7 / 12 \log _{2}(7 / 12)$ bits, and each individual symbol gives an entropy of $-1 / 12 \log _{2}(1 / 12)$ bits. For an individual symbol we can write $-1 / 12 \log _{2}(1 / 12)=1 / 12 \log _{2}(12)$, because $\log (1 / x)=-\log (x)$.
Example 1

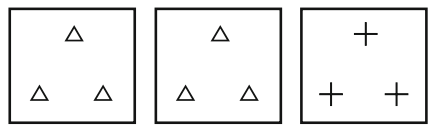

Entropy $=0.92$

Example 2

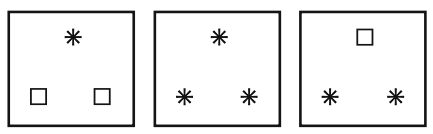

Entropy $=0.92$

Example 3

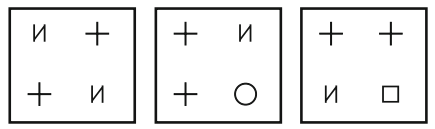

Entropy $=1.63$

Example 4

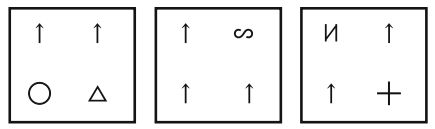

Entropy $=1.95$

Example 5
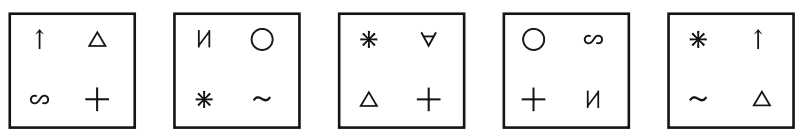

Entropy $=3.11$

Figure 1. Five examples of discrete oddity problems, with their entropy levels. 
$1 / 12 \log _{2}(12)$ indicates that in order to code 12 different discrete symbols, $\log _{2}(12)$ bits of information are needed and that one such symbol represents $1 / 12$ of that amount of information.

We then compute the entropy score of every individual subject in the present study by summing the entropy values of every item correctly answered by that subject.

\section{Analysis \\ To test our core hypothesis, we used SPSS 16.0 and performed the following four distinct analyses: (1) a correlation analysis of the four CFIT subtests - series, classification, matrices, conditions - together with the R-ASCM entropy; (2) a principal component analysis of the four CFIT subtests only; (3) a principal component analysis of the four CFIT subtests with the R-ASCM entropy included; and (4) a correlation analysis using Spearman's rho to establish the link between the R-ASCM items' pass rate and their level of entropy.}

\section{RESULTS}

\section{Analysis 1}

Table 1 presents the descriptive statistics and a correlation matrix for all the variables used in the study. The results show that, as expected, classification has the highest statistically significant coefficient of correlation with the $\mathrm{R}$-ASCM entropy. This is the highest correlation in the matrix by far, which seems to indicate that the two tests measure something in common. Another notable aspect of the correlation table is that the magnitudes of the correlations among the four subtests of the CFIT are not very high, even if most of the coefficients are statistically significant. The highest correlation among the four CFIT subtests is between classification and matrices; conditions, however, is only significantly correlated with classification within the CFIT. The R-ASCM entropy is significantly correlated with three of the four CFIT subtests (classification, matrices, and conditions) but not with series.

\section{Analysis 2}

Table 2 reports the results of the principal component analysis of the four CFIT subtests. To ensure suitability for conducting factor analysis, the Kaiser-Mayer-Olkin (KMO) test and Bartlett's test of sphericity were used. The KMO statistic (see Kaiser, 1970) can be calculated for individual and multiple variables and represents the ratio of the squared correlation between variables to the squared partial correlation between variables - it is a measure of shared variance. Kaiser (1974) recommended accepting values greater than 0.5 as acceptable. The result of the global KMO test for this analysis is 0.61 . The KMO values for individual variables were produced on the diagonal of the anti-image correlation matrix and they all range between 0.59 and 0.65 ; therefore, none of the variables should be excluded from the analysis. Bartlett's test of sphericity determines whether the correlation matrix is an identity matrix - that is, whether all variables are uncorrelated. If an identity matrix exists, factor analysis is meaningless. The result of Bartlett's test of sphericity is relatively high at $32(p<.001)$.

To determine the number of factors, both the eigenvalues (Kaiser's criterion) approach and the Scree test approach were used. Both approaches gave convergent results and only one component emerged with an eigenvalue of 1.5 , explaining $38 \%$ of the total variance. Since only one com-
Table 1

\begin{tabular}{lrrrrrr}
\multicolumn{7}{c}{ Descriptive Statistics and Correlations } \\
\hline \multicolumn{1}{c}{ Variables } & \multicolumn{1}{c}{$M$} & $S D$ & 1 & 2 & 3 & 4 \\
\hline Series & 8.69 & 1.28 & & & & \\
Classification & 10.38 & 1.40 & $.17^{*}$ & & & \\
Matrices & 7.51 & 1.34 & $.19^{* *}$ & $.26^{* *}$ & & \\
Conditions & 8.59 & 1.38 & .07 & $.21^{* *}$ & .10 & \\
R-ASCM entropy & 67.36 & 7.15 & .09 & $.43^{* *}$ & $.17^{* *}$ & $.21^{* *}$ \\
\hline
\end{tabular}

${ }^{*} p<.05 . \quad{ }^{* *} p<.01$

ponent was extracted, no rotation was performed. This first and unique principal component is the general factor of the CFIT and is relatively large. Because the CFIT is mostly a measure of fluid intelligence, the first component can be considered to be the $g$ factor, or a very similar construct, and we will refer to it below as $g$. The subtest with the highest $g$ loading is classification, followed by matrices. Conditions, however, has a loading on $g$ lower than .50 , which concurs with the low correlations already observed.

\section{Analysis 3}

Table 3 reports the results of the principal component analysis of the four CFIT subtests with the R-ASCM entropy. To ensure suitability for conducting factor analysis, the KMO test and Bartlett's test of sphericity were used. The result of the global KMO test for this analysis is 0.65 . The KMO values for individual variables all range between 0.62 and 0.75 , so none of the variables should be excluded from the analysis. The result of Bartlett's test of sphericity is quite high at $71(p<.001)$.

To determine the number of factors, both the eigenvalues (Kaiser's criterion) approach and the Scree test approach were used. Both approaches gave convergent results, and again, only one component emerged with an eigenvalue of 1.8 , explaining $36 \%$ of the total variance. Since only one component was extracted, no rotation was performed. This first and unique principal component is comparable to the general factor of the CFIT in terms of percentage of variance explained and subtests loadings. The first component can thus be considered again to be

Table 2

Principal Components Matrix for the CFIT Subtests

\begin{tabular}{lc}
\hline Variables & $g$ Loadings \\
\hline Series & .56 \\
Classification & .72 \\
Matrices & .67 \\
Conditions & .49 \\
\% Variance & 38 \\
\hline
\end{tabular}

Table 3

Principal Components Matrix for the CFIT Subtests and the $\mathrm{R}-\mathrm{ASCM}$

\begin{tabular}{lc}
\multicolumn{1}{c}{ Variables } & $g$ Loadings \\
\hline Series & .42 \\
Classification & .77 \\
Matrices & .56 \\
Conditions & .48 \\
R-ASCM entropy & .70 \\
\% Variance & 36 \\
\hline
\end{tabular}


the $g$ factor, or a very similar construct, and we will refer to it below as $g$. The test with the highest $g$ loading is classification, immediately followed by the R-ASCM entropy. The test with the lowest $g$ loading is series, with a loading lower than .50 .

Conclusion 1. The R-ASCM entropy score, which is measured on a ratio scale, mostly loads $g(.70, p<.001)$. Construct validity is therefore established and the main hypothesis is strongly supported.

\section{Analysis 4}

This supplementary analysis was conducted to confirm Conclusion 1 above from the different perspective of item response theory. Because entropy is essentially a property of the R-ASCM items, there should be a direct relationship between the items' pass rate (their difficulty level) and their level of entropy (their complexity level). However, difficulty of an item (proportion passing) is specific to a particular group of persons to whom the test is administered, and as such it cannot be considered an intrinsic attribute of the test items. In our context, therefore, a percentage of variance in entropy explained by "difficulty" (or vice versa) would have little meaning, and instead of Pearson's $r$, Spearman's rho, a robust nonparametric coefficient of correlation used for ranks, was preferred. For the 35 items of the R-ASCM a rho of $-.74(p<.001)$ between their entropy rank and their proportion passing rank was obtained. Spearman's rho between the proportion passing rank and the subjective rank order of the test items (the order in which the items appear on the test, on the basis of my subjective idea of increasing difficulty) was $-.77(p<.001)$. Finally, rho between the order in which the items appear on the test and their entropy rank was .75 $(p<.001)$.

Conclusion 2. The relationship between the R-ASCM items' complexity rank and their difficulty rank is high and very significant $(-.74, p<.001)$, which indicates that the difficulty of the R-ASCM is mostly due to its complexity or entropy level.

\section{DISCUSSION}

The primary aim of this research was to devise a vehicle that would most accurately and efficiently measure an individual's standing on the $g$ construct. For that purpose, two measures of nonverbal inductive reasoning, the CFIT and the R-ASCM, were used. This study reveals that it is possible to measure the $g$ factor on a ratio scale of complexity if the proper vehicle is used (e.g., the R-ASCM). Because $g$ is manifested in so many kinds of behavior that involve mental abilities, an individual's level of $g$ can be elicited in many different ways and the R-ASCM entropy is only one possible ratio scale of intelligence.

An important characteristic of our sample is the highly restricted ability range of students. All the French ESCs are extremely competitive schools and the mean raw score of our sample on the CFIT is very high at 35.16 (out of a maximum possible score of $50, S D=3.31$ ). Detterman and Daniel (1989) were the first to systematically and convincingly study the phenomenon known as the "law of diminishing returns," originally discovered by Spearman. That is, the higher the level of $g$, the less is the amount of $g$ variance in any particular mental test. We can therefore expect lower correlations in our sample than for the general population; indeed, $g$ accounts for less than $40 \%$ of total variance in the correlation matrix. Nevertheless, the results of the principal component analysis are unambiguous.

When discussing factor analytic results and reporting the proportion of variance explained, it is essential to be clear about what type of analysis (principal components or common factors) and thus what type of variance (shared or total) is being explained (DeVellis, 2003). Components account for a specified portion of the total variance among the original variables, whereas factors account for the shared variance only. Factors explain a larger proportion of a more restricted variance (common variance), whereas components explain a larger proportion of total variance. From the perspective of factor analysis, unshared variation is essentially error variance, because factors are supposed to determine how items are answered. But from the perspective of principal components, the components are defined by how the items are answered; the components are end products of the items, and the actual scores obtained on items determine the nature of the components.

The present study does not hypothesize error-free underlying variables or factors; its main purpose is to establish how entropy is structurally related to the CFIT. Because entropy is a ratio scale measured in bits, the present research does not assume that the variance that entropy does not share with the CFIT is simply error variance.

\section{Practical and Theoretical Implications}

The R-ASCM entropy score is a ratio scale because it has a true zero point ( 0 bit) and because units of measurement (bits) represent equal intervals of the amount of information processed by the subjects. The average score of the sample is 67.4 bits and the $S D$ is 7.15 bits or $7.15 / 67.4=11 \%$ of the average score (see Table 1 ). (The $S D$ is a measure of spread, which roughly indicates the average deviation from the mean.) The lowest score on the R-ASCM is 50.4 bits and the highest score is 79.3 bits, which indicates that the best subject managed efficiently $79.3 / 50.4=1.57$, or $57 \%$ more information than the worst subject did.

At this stage, the effect of the time limit (15 min) on the standard version of the R-ASCM is unknown. We do not know for sure whether the majority of subjects reached their own ceiling of difficulty, even if it is likely. One simple way to resolve this matter would be to administer both the standard and the advanced versions of the R-ASCM as power tests. The advanced version contains many difficult items; what is needed is to let the subjects reach their ceiling of difficulty - that is, the entropy of the hardest questions they can answer correctly and the true level of complexity they can manage.

The meaning of a ratio scale must be further explained. Ratio scales are mostly used in physics to measure concepts such as length, mass, or time; but ratio scales are very rare in psychological measurement, since meaningful zero points are hard to find. For example, most physi- 
cal measures such as weight or height qualify, and perhaps some physiological measures such as electrodermal response.

The R-ASCM is a test of nonverbal inductive reasoning. All inductive-reasoning tasks share the same basic property: A set of stimuli is presented and the purpose is to infer the pattern or rule structure for the item. Entropy measures the complexity of the set of stimuli (or of the rule structure) for every test item, as a ruler measures the length of an object. Entropy itself has nothing to do with the test takers; it is an exclusive property of the test items, and traditional psychometric measures, such as coefficient $\alpha$, are ultimately irrelevant.

Entropy exists independently of human beings and it has the same importance here as do degrees Kelvin for the measurement of temperature. Most people can feel external temperature, and a factor analysis of human perceptions would most likely indicate a "heat factor." High scorers would be described as more sensitive to heat, whereas low scorers would be described as less sensitive. But it would occur to nobody that a score on such a factor should be more important than the temperature obtained from a thermometer in degrees Kelvin. A very elaborate theory of the heat construct as felt by human beings could also be developed. Nevertheless, limiting heat itself to this heat perception (or heat factor) would be a serious obstacle; in such a context, meteorology or thermodynamics could never appear. Similarly, preferring to limit complexity itself, as measured by entropy, to the complexity factor (or the $g$ factor) is like preferring the shadow of reality to reality itself: scientifically questionable.

Together with the classification subtest, the R-ASCM is the best measure of inductive reasoning available in this study. When the four subtests of the CFIT are factor analyzed without the R-ASCM, classification is still the best measure of inductive reasoning available, better than matrices (a type of test invented by Spearman and mostly developed by Raven). It may be because finding the "odd man out" is of more fundamental importance for the measurement of intelligence than what is measured by the other CFIT subtests. Thomas Aquinas (1225-1274) defined intelligence as "the ability to see the similarity among dissimilar things and the dissimilarities among similar things," which is a fair characterization of what takes place in the R-ASCM or the classification subtest. Jensen (1980) reports that numerous ingenious tests have been devised to compare the behavioral capacities of animals, and that a popular type of learning set is precisely the oddity problem (sometimes referred to as "match-tosample" problems in the animal literature). The animal is offered three (or more) stimulus objects, two alike and one different - for example, two triangles and a square. Response to the odd one is always rewarded. Improvement in performance indicates that the animal is grasping the abstract concept of oddity. On the other hand, it seems quite impossible to measure a less-than-human intelligence with a matrix test, or with any of the Wechsler subtests, which seems to indicate once more that the oddity problems measure a more fundamental process than do most of the other assessments of inductive reasoning currently available.

It is because the environment we live in is complex, in the information theory sense, that we can measure $g$ with entropy. Entropy can measure the complex information processing demand of some essential aspects of the world in which the human brain has appeared, and insofar as $g$ reflects the ability to deal with this complexity (Cattell, 1940; Gottfredson, 1997; Heylighen, 1994; Jensen, 1980), $g$ is a consequence of or an adaptation to entropy.

At the level of complex psychometric tests the $g$ factor is unitary, but it now seems very unlikely that $g$ is unitary at the level of its causal underpinnings (Jensen, 1998). The brain is structurally and functionally the most complex organ of the human body, and the greater part of this complexity resides in the neural structure of the neocortex. Crinella and Yu (1995) have supported the theory that tests with higher $g$ loadings involve proportionately more neural processes than do tests with lower $g$ loadings, even when studied in nonhuman animals. Thus, it seems currently difficult to discover in the human brain one simple biological origin of $g$ and to use it as the sole basis of a theoretical superstructure of intelligence. Moreover, we cannot rule out that machines or some alien species may one day develop high intelligence - the ability to deal with high complexity - without any kind of $g$ (as rockets can fly without wings). This hypothesis, of course, is impossible to test, but we can already be quite confident that if such a different kind of intelligence exists, entropy will remain a relevant ratio scale of measurement (McCowan, Doyle, \& Hanser, 2002).

In conclusion, the most important contribution of this research is that the complexity of some fluid intelligence tests can be measured on a ratio scale - a scale with a true zero and equal intervals. The name of this measure is entropy, a tool traditionally used for data compression. Entropy can quantify with great accuracy the amount of information contained in a range of nonverbal inductive reasoning tests.

\section{AUTHOR NOTE}

Correspondence concerning this article should be addressed to P. E. Ruiz, ESC Lille, Av. Willy Brandt, 59777 Euralille, France (e-mail: p.ruiz@esc-lille.fr).

\section{REFERENCES}

BARretT, P. (2000). Intelligence, psychometrics, IQ, g, and mental abilities: Quantitative methodology dressed as science. Psycoloquy, 11. Available at www.cogsci.ecs.soton.ac.uk/cgi/psyc/newpsy?11.046

Campbell, D. J. (1988). Task complexity: A review and analysis. Academy of Management Review, 13, 40-52.

Cattell, R. B. (1940). A culture free intelligence test, Part I. Journal of Educational Psychology, 31, 161-169.

Cattell, R. B. (1941). Some theoretical issues in adult intelligence testing [Abstract]. Psychological Bulletin, 38, 592.

CAttell, R. B. (1971). Abilities: Their structure, growth, and action. Boston: Houghton Mifflin.

Crinella, F. M., \& YU, J. (1995). Brain mechanisms in problem solving and intelligence: A replication and extension. Intelligence, $\mathbf{2 1}$ $225-246$

Detterman, D. K., \& Daniel, M. H. (1989). Correlates of mental tests 
with each other and with cognitive variables are highest for low IQ groups. Intelligence, 13, 349-359.

DeVellis, R. F. (2003). Scale development: Theory and application (2nd ed.). Thousand Oaks, CA: Sage.

FLYNN, J. R. (1987). Massive IQ gains in 14 nations: What IQ tests really measure. Psychological Bulletin, 101, 171-191.

Goodman, N. (1972). Seven strictures on similarity. In N. Goodman (Ed.), Problems and projects (pp. 437-446). Indianapolis: Bobbs-Merrill.

GotTFREDSON, L. S. (1997). Why $g$ matters: The complexity of everyday life. Intelligence, 24, 79-132.

Gregory, R. J. (2004). Psychological testing: History, principles, and applications. Needham Heights, MA: Allyn \& Bacon.

Halford, G. S., BaKer, R., McCredden, J. E., \& Bain, J. D. (2005). How many variables can humans process? Psychological Science, 16, 70-76.

Halford, G. S., Wilson, W. H., \& Phillips, W. (1998). Processing capacity defined by relational complexity: Implications for comparative, developmental and cognitive psychology. Behavioral \& Brain Sciences, 21, 803-864.

Heylighen, F. (1994). Fitness as default: The evolutionary basis for cognitive complexity reduction. In R. Trappl (Ed.), Cybernetics and Systems '94 (pp. 1595-1602). Singapore: World Science.

HochberG, J., \& MCAlister, E. (1953). A quantitative approach to figural "goodness." Journal of Experimental Psychology, 46, 361-364.

Horn, J. L. (1968). Organization of abilities and the development of intelligence. Psychological Review, 75, 242-259.

HorN, J. L. (1985). Remodeling old models of intelligence. In B. B. Wolman (Ed.), Handbook of intelligence: Theories, measurements, and applications. New York: Wiley.

IPAT (1973a). Measuring intelligence with the culture fair tests: Manual for scales 2 and 3. Champaign, IL: Institute for Personality and Ability Testing.

IPAT (1973b). Technical supplement for the culture fair intelligence tests: Scales 2 and 3. Champaign, IL: Institute for Personality and Ability Testing.
Jensen, A. R. (1980). Bias in mental testing. New York: Free Press.

Jensen, A. R. (1998). The g factor: The science of mental ability. Westport, CT: Greenwood.

JenSEN, A. R. (2006). Clocking the mind: Mental chronometry and individual differences. Amsterdam: Elsevier.

KaIser, H. F. (1970). A second-generation little jiffy. Psychometrika, 35, 401-415.

KaISer, H. F. (1974). An index of factorial simplicity. Psychometrika, 39, 31-36.

McCowan, B., Doyle, L. R., \& Hanser, S. F. (2002). Using information theory to assess the diversity, complexity, and development of communicative repertoires. Journal of Comparative Psychology, 116 166-172.

NARENS, L. (1985). Abstract measurement theory. Cambridge, MA: MIT Press.

Petersen, N. S., Kolen, M. J., \& Hoover, H. D. (1989). Scaling, norming, and equating. In R. L. Linn (Ed.), Educational measurement (3rd ed.). New York: Macmillan.

Shannon, C. E. (1948). A mathematical theory of communication. Bell System Technical Journal, 27, 379-423, 623-656.

Spearman, C. (1904). "General intelligence," objectively determined and measured. American Journal of Psychology, 15, 201-293.

SPEARMAN, C. (1923). The nature of "intelligence" and the principles of cognition. London: Macmillan.

Stevens, S. S. (1946). On the theory of scales of measurement. Science, 103, 677-680.

Wicherts, J. M., Dolan, C. V., Hessen, D. J., Oosterveld, P., van BaAl, G. C. M., Boomsma, D. I., \& Span, M. M. (2004). Are intelligence tests measurement invariant over time? Investigating the nature of the Flynn effect. Intelligence, 32, 509-537.

(Manuscript received January 21, 2009; accepted for publication February 19, 2009.) 